\title{
Pteridófitas da Serra Negra, Minas Gerais, Brasil
}

Filipe Soares Souza ${ }^{1,4}$, Alexandre Salino², Pedro Lage Viana3 e Fátima Regina Gonçalves Salimena ${ }^{1}$

Recebido em 7/07/2011. Aceito em 24/02/2012

\section{RESUMO}

(Pteridófitas da Serra Negra, Minas Gerais, Brasil). Este trabalho apresenta uma lista de espécies de pteridófitas que ocorrem na Serra Negra, Minas Gerais. A serra está inserida no complexo da Serra da Mantiqueira situada entre Rio

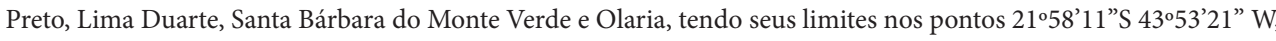

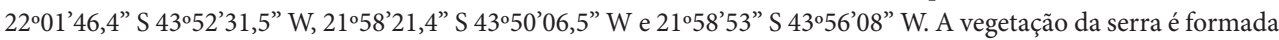
por um mosaico de fitofisionomias, sendo encontradas formações florestais (florestas ombrófilas e semidecíduas) e campestres (campos rupestres). O inventário florístico foi realizado entre os anos de 2003 e 2008, em excursões mensais para coleta de amostras e registro de dados. Na serra foram registradas 209 táxons infragenéricos distribuídas em 24 famílias e 75 gêneros. As famílias com maior número de espécies foram Polypodiaceae (40), Dryopteridaceae (33) e Pteridaceae (25). A maioria das espécies (109) foi encontrada ocorrendo exclusivamente no interior de floresta. Em relação ao hábito, 69 espécies foram encontradas exclusivamente como terrestres, 37 como rupícolas ou terrestres e 32 exclusivamente epífitas. Este trabalho revela uma elevada riqueza de pteridófitas na região e indica a importância de estudos desta natureza na conservação e manejo das pteridófitas em Minas Gerais.

Palavras-chave: Florística, Licófitas, Mata Atlântica, Minas Gerais, Samambaias

\begin{abstract}
(Ferns and lycophytes of Serra Negra, Minas Gerais, Brazil). This work presents an inventory of pteridophyte species that occur in the Serra Negra, of Minas Gerais. The area is part of the Mantiqueira Range, and is situated between

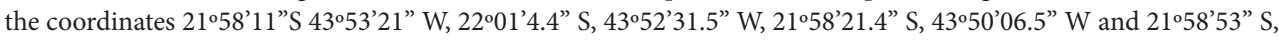
$43^{\circ} 56^{\prime} 08^{\prime \prime} \mathrm{W}$. The vegetation in the Serra Negra is characterized by a mosaic of different phytophysiognomies, divided into forests (evergreen and seasonal semideciduous forests) and open formations (rocky grasslands). The inventory was carried out from 2003 to 2008, during monthly excursions to collect botanical material and data in the study area. A total of 209 species, distributed in 24 families and 75 genera, were recorded. The families with the highest number of species were Polypodiaceae (40), Dryopteridaceae (33) and Pteridaceae (25). More than the half (110) of the inventoried species were recorded exclusively in the forests formations. Sixty-nine species were terrestrial, 37 were saxicolous or terrestrial and 32 were epiphytes. This work shows that this region is rich in ferns and reveals the importance of this kind of study for conservation and management of pteridophytes in the state of Minas Gerais.
\end{abstract}

Key words: Atlantic Forest, Ferns, Floristics, Lycophytes, Minas Gerais

\section{Introdução}

As pteridófitas constituem um importante componente da flora de florestas tropicais úmidas, compreendendo geralmente cerca de $10 \%$ do total do número de espécies de plantas vasculares (Grayum \& Churchill 1987). Esse grupo de plantas ocorre em diversos ecossistemas, apresentando representantes em regiões ártico-alpinas, áreas subdesérticas, interior de florestas tropicais úmidas, regiões rochosas costeiras e mangues (Page 1979). Na região Sudeste do
Brasil, são encontradas cerca de 790 táxons infraespecíficos de pteridófitas, sendo que a maioria ocorre nas florestas úmidas da Serra do Mar (Prado \& Sylvestre 2011; Tryon \& Tryon 1982).

Segundo Moran (1995), as montanhas influenciam a distribuição e a diversidade de pteridófitas, impedindo a migração e promovendo um alto grau de endemismo e riqueza especifica. A Floresta Atlântica é um notável centro de endemismo na região Neotropical (Tryon 1972), integrando um dos quatro maiores hot spots mundiais de

\footnotetext{
1 Universidade Federal de Juiz de Fora, Departamento de Botânica, Instituto de Ciências Biológicas, Juiz de Fora, MG, Brasil

2 Universidade Federal de Minas Gerais, Departamento de Botânica, Instituto de Ciências Biológicas, Belo Horizonte, MG, Brasil

Jardim Botânico Inhotim, Brumadinho, MG, Brasil

4 Autor para correspondência: ssepilif@yahoo.com.br
} 
biodiversidade, com $40 \%$ das espécies endêmicas desse bio$\mathrm{ma}$ (Myers et al. 2000). Atualmente, esse bioma é altamente ameaçado, principalmente pelos constantes desmatamentos, seus poucos remanescentes encontram-se fragmentados e em boa parte são constituídos por florestas secundárias (Mynssen \& Windisch 2004). A região da Serra da Mantiqueira apresenta uma alta riqueza de espécies de fauna e flora, especialmente no que se trata de espécies raras, endêmicas e ameaçadas (Drummond et al 2005). Porém a carência de inventários biológicos em muitas regiões da Serra da Mantiqueira impossibilita a avaliação das áreas em relação indicação de ações concretas para conservação da biodiversidade existente nessas áreas.

Para a Floresta Atlântica na região Sudeste do país, alguns autores se dedicaram aos estudos de ocorrência de pteridófitas. Entre os principais trabalhos florísticos realizados destacam-se: Brade (1948), Melo \& Salino $(2002,2007)$, Prado \& Labiak (2003), Mynssen \& Windisch (2004), Santos et al. (2004), Dittrich et al. (2005), Figueredo \& Salino (2005), Sakagami (2006), Condack (2006), Santos \& Sylvestre (2006), Schwartsburd \& Labiak (2007), Boldrin \& Prado (2007), Salino \& Almeida (2008a) e Salino \& Almeida (2009).

Para o estado de Minas Gerais existem poucos trabalhos sobre levantamento de pteridófitas, alguns apresentam listas de espécies (Brade 1942, 1949; Melo \& Salino 2002, 2007; Figueiredo \& Salino 2005), ou tratamentos taxonômicos, contudo muitos são restritos a uma família e a maior parte destes, restritos à Cadeia do Espinhaço (Carvalho 1982; Camargo 1983; Windisch \& Prado 1990; Prado 1992, 1997; Windisch 1992a; Prado \& Windisch 1996; Rolim 2007; Salino \& Almeida 2008b, Salino \& Garcia 2008; Rolim \& Salino 2008; Viveiros 2010); Assis \& Salino (2011). No complexo da Serra da Mantiqueira, Melo \& Salino (2007) apresentaram uma listagem de pteridófitas ocorrentes na APA Fernão Dias, região de Camanducaia e Gonçalves e Condack (2006), apresenta uma listagem das regiões altomontanas do Parque Nacional do Itatiaia. Próximo à área desse estudo, no Parque Estadual do Ibitipoca, Novelino \& Oliveira (1999) realizaram um estudo do gênero Elaphoglosum e, posteriormente, Salino et al. (dados não publicados) inventariaram as espécies de pteridófitas nesta unidade de conservação. Na área de estudos apenas o levantamento feito por Menini Neto et al. (2009) trata de pteridófitas, porém o foco deste são plantas vasculares não-arbóreas em uma pequena localidade da Serra Negra.

O objetivo deste trabalho foi inventariar as espécies de pteridófitas ocorrentes na Serra Negra (complexo da Serra da Mantiqueira), no estado Minas Gerais e estabelecer as relações de similaridade florística desta com outras áreas da Floresta Atlântica.

\section{Material e métodos}

A Serra Negra está inserida no complexo da Serra da Mantiqueira, situada nos municípios de Rio Preto, Lima Duarte, Santa Bárbara do Monte Verde e Olaria, tendo seu limite Norte no ponto $21^{\circ} 58^{\prime} 11^{\prime \prime} S 43^{\circ} 53^{\prime} 21^{\prime \prime}$ W, Sul

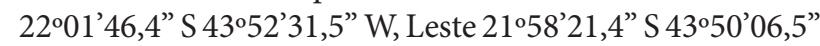
W e Oeste $21^{\circ} 58^{\prime} 53^{\prime \prime}$ S $43^{\circ} 56^{\prime} 08^{\prime \prime}$ W, perfazendo uma área de aproximadamente 10000 hectares (Fig. 1). Na região são encontradas duas Unidades de Conservação em processo de formação: Reserva Particular de Patrimônio Natural (RPPN) São Lourenço do Funil e RPPN Serra Negra.

O clima é do tipo Cwb (Köppen, 1931), mesotérmico úmido e a precipitação anual de $1886 \mathrm{~mm}$. Na área são encontradas elevações de 900 a 1698 m, o que propicia um mosaico de vegetação associado a diferentes condições climáticas e edáficas.

Observou-se na área uma clara distinção de fitofisionomias em função do gradiente altitudinal, fatores edáficos e disponibilidade hídrica. Nas cotas altidutinais inferiores (entre aproximadamente 800 e $1100 \mathrm{~m}$ ), são encontradas florestas aluviais, alagáveis, localmente conhecidas como "Cambuí" e caracterizadas pela monodominância da espécie arbórea Myrciaria tenella (Myrtaceae); nessas cotas altitudinais ocupando encostas de morros é encontrada a Floresta Estacional Semidecidual; ao longo das margens de riachos, apresenta caráter mais perenifólio, com árvores de grande porte e maior abundância de epífitas,; onde há afloramentos de rochas quartzíticas, com solo arenoso, há Campos Rupestres, com extrato arbustivo dominante. Em direção aos topos dos morros, com o incremento de altitude, as formações florestais tendem a apresentar menor estatura $\mathrm{e}$ abundância expressiva de epífitas, caracterizando a Floresta Ombrófila Densa Altomontana (ou “matas nebulares") típicas de altitudes superiores a $1500 \mathrm{~m}$ na região; nas cumeeiras, a vegetação associada os afloramentos quartzíticos apresenta o estrato herbáceo mais contínuo, coberto por capins e pequenos arbustos.

Os trabalhos de campo para coleta de amostras e registro de dados foram realizados entre os anos de 2003 e 2008. Os vários ambientes foram explorados na área de estudos através do Método do Caminhamento (Filgueras et al. 1994). As amostras em estado fértil foram coletadas e preparadas segundo técnicas usuais utilizadas para pteridófitas (Silva 1989) e posteriormente depositadas nos acervos dos herbários

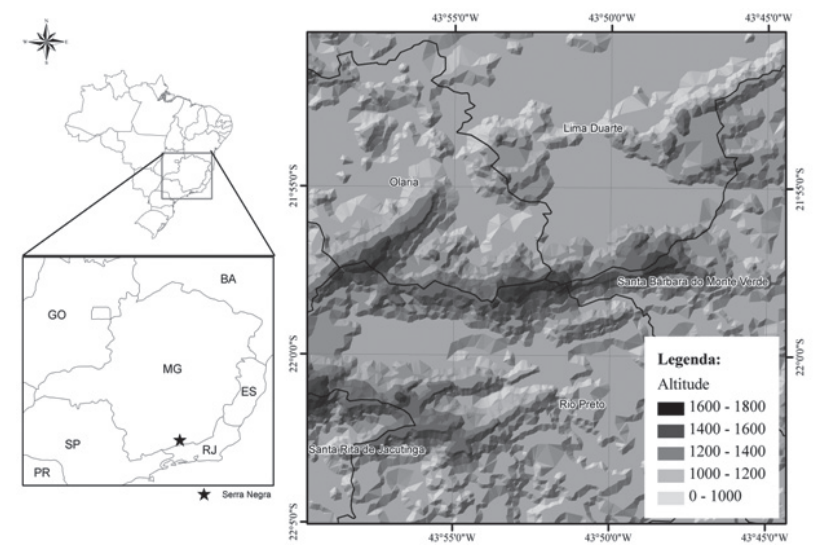

Figura 1. Localização da Serra Negra. 
Leopoldo Krieger, da Universidade Federal de Juiz de Fora (CESJ) e da Universidade Federal de Minas Gerais (BHCB).

A identificação das espécies foi realizada com auxílio de literatura especializada e por comparação com material já determinado por especialistas, existentes nos herbários CESJ, BHCB e RB. O sistema de classificação usado para a listagem dos táxons foi o de Smith et al. (2006a) para as monilófitas e Kramer \& Green (1990) para as licófitas. A abreviatura dos autores dos táxons seguiu Pichi Sermolli (1996). Dados referentes aos status de conservação das espécies para o estado de Minas Gerais foram retiradas de Drummond et al (2008) e para o Brasil de Ministério do Meio Ambiente (2008).

Para análise das relações de similaridade florística entre a área estudada e outras 13 áreas das cadeias da Mantiqueira e Espinhaço (Tab. 2) foi utilizado o coeficiente de similaridade de Sørensen (Magurran 2005) e o algorítimo WPGMA, para evitar que diferenças no esforço amostral venham interferir na análise (Sokal \& Michener 1958).

\section{Resultados e discussão}

$\mathrm{Na}$ área foram coletados 621 espécimes de pteridófitas, distribuídos em 24 famílias, 75 gêneros e 209 táxons infragenéricos. (Tab. 1). O número de táxons infragenéricos encontradas destaca a grande riqueza de pteridófitas na área de estudos. Uma comparação com outras áreas inventariadas na região sudeste/sul do Brasil (Tab. 2) revela a Serra Negra dentre as áreas com maior número de espécies para as Regiões Sul e Sudeste do país.

As famílias com maior número de táxons infragenéricos foram Polypodiaceae (40 spp.), Dryopteridaceae (33), Pteridaceae (25), Aspleniaceae (19) e Hymenophyllaceae (14). Os gêneros mais bem representados foram Elaphoglossum (20), Asplenium (19), Blechnum (12), Thelypteris (8), Hymenophyllum (8) e Doryopteris (8). A grande maioria dos gêneros encontrados $(41,5 \%)$ é representada por um a quatro táxons infragenéricos.

Alguns táxons infragenéricos foram encontradas apenas nas áreas mais altas da serra (acima de 1200 m.s.m.), como Asplenium harpeodes, A. incurvatum, A. pseudonitidum, Blechnum binervatum subsp. acutum, B. schomburgkii, Ceradenia albidula, Culcita coniifolia, Dicksonia sellowiana, Doryopteris rosenstockii, D. rufa, Elaphoglossum langsdorffi, Grammitis fluminensis, Huperzia pungentifolia e Zygophlebia longipilosa. Entre os táxons que foram registrados exclusivamente nas áreas mais baixas (abaixo de 1000 m.s.m.), destacam-se Adiantopsis radiata, Asplenium kunzeanum, A. martianum, A. flabellulatum, Danaea geniculata, Didymochlaena truncatula, Microgramma tecta e Stigmatopteris caudata. Estes dados refletem a grande heterogeneidade de hábitats na área de estudos, onde são encontradas diversas fitofisionomias em função do gradiente altitudinal, um fator responsável pela expressiva diversidade de pteridófitas registrada. $\mathrm{Na}$ área foram encontradas quatro espécies exóticas ou subespontâneas: Deparia petersenii, Macrothelypteris torresiana, Pteris vittata e Thelypteris dentata.

Em relação ao ambiente de ocorrência dos táxons infragenéricos, observou-se que 110 espécies $(53,14 \%)$ ocorrem exclusivamente em ambientes florestais, seguido de 25 $(12,08 \%)$ que se encontram ou no campo rupestre ou em florestas, 13 são encontradas em três ambientes diferentes: Anemia raddiana, Blechnum cordatum e Dicranopteris flexuosa encontrados no campo rupestre, borda de mata e área antropizada, Elaphoglossum luridum, Phlebodium pseudoaureum, Pleopeltis hirsutissima, Pleopeltis macrocarpa, Serpocaulon catharinae, Serpocaulon latipes e Sphaeropteris gardneri ocorrem no campo rupestre, borda e interior de florestas, Thelypteris dentata foi encontrado em área antropizada e borda e interior de florestas, Elaphoglossum lingua e Lycopodiella cernua foram observadas no campo rupestre, interior de floresta e área antropizada, os táxons infragenéricos Doryopteris collina, Lycopodium clavatum e Sticherus bifidus foram encontradas em quatro ambientes diferentes (campo rupestre, borda de floresta, interior de floresta e área antropizada).

Quanto ao hábito, 69 táxons infragenéricos foram encontradas exclusivamente como terrestres, seguida de 37 rupícolas ou terrestres e 32 exclusivamente epífitas. Dez táxons infragenéricos foram observadas como rupícola, terrestre ou epífita (Campyloneurum acrocarpon, C. nitidum, Elaphoglossum pachydermum, Hymenophyllum polyanthos, Melpomene pilosissima, Phlebodium pseudoaureum, Pleopeltis hirsutissima, Polyphlebium angustatum, Serpocaulon latipes e Selaginella decomposita), apenas duas táxons infragenéricos (Lygodium volubile e Salpichlaena volubilis) são plantas terrestres com frondes escandentes, Blechnum organense, Mickelia guianensis e Polybotrya speciosa apresentam-se como hemiepífitas exclusivamente.A ocorrência de pteridófitas epífitas na área é baixa (15,46\%), quando comparada com outros estudos em florestas ombrófilas na Serra do Mar, como Sylvestre (1997a), que encontrou cerca de $48 \%$ de epífitas para uma área no estado do Rio de Janeiro e Dittrich et al. (2005), que encontrou cerca de $62 \%$ para uma área no Paraná. Essa diferença de valores pode ser explicada pela existência, na área coletada, de um mosaico vegetacional, formado por Floresta Estacional Semidecidual, Floresta Ombrófila, além de Campos Rupestres entremeados a estas florestas. Desses ambientes, alguns são menos propícios à ocorrência de epífitas, como as Florestas Estacionais Semideciduais e Campos Rupestres. Porém, quando comparada a áreas de Floresta Estacional Semidecidual no estado de Minas Gerais, como no vale do rio Doce (Melo \& Salino 2002), que apresenta cerca de 8\% de espécies epífitas, e na APA-Sul RMBH (Figueredo \& Salino 2005) 13,7\% de epífitas, observamos um índice maior na área de coleta, este fato também pode ser explicado pela existência desse mosaico vegetacional, pois a existência de áreas como Florestas Ombrófilas possibilitam uma maior ocorrência de epífitas. 
Tabela 1. Lista dos táxons ocorrentes na Serra Negra com hábito e ambiente encontrados, estado de conservação no Brasil e Minas Gerais e distribuição geográfica. Legenda: Habitat: AR (Arborescente); TE (Terrestre); EP (Epífita); RU (Rupícola); SX (Saxícola); LI (Liana); HE (Hemi-epífita); Ambiente: IM (Interior de mata); CR (Campo Rupestre); BM (Borda de mata); AA (Área antropizada); BR (Brejo).

\begin{tabular}{|c|c|c|c|}
\hline Táxon & Hábito & Ambiente & Material Examinado \\
\hline \multicolumn{4}{|l|}{ Anemiaceae } \\
\hline Anemia oblongifolia (Cav.) Sw. & $\mathrm{RU} / \mathrm{TE}$ & $\mathrm{CR} / \mathrm{IM}$ & Souza 11 \\
\hline A.phyllitidis (L.) Sw. & $\mathrm{TE}$ & $\mathrm{BM} / \mathrm{IM}$ & Souza 523 \\
\hline A. raddiana Link. & $\mathrm{RU} / \mathrm{TE}$ & $\mathrm{CR} / \mathrm{BM} / \mathrm{AA}$ & Souza 23 \\
\hline A. villosa Humb. \& Bonpl. ex Willd. & RU & $\mathrm{CR} / \mathrm{BM}$ & Souza 708 \\
\hline Anemia sp. 1 & RU & CR & Souza 90 \\
\hline Anemia sp. 2 & EP & IM & Salimena 2693 \\
\hline \multicolumn{4}{|l|}{ Aspleniaceae } \\
\hline Asplenium alatum Humb. \& Bonpl. ex Willd. & RU/TE & $\mathrm{IM}$ & Souza 129 \\
\hline A. auriculatum Sw. & $\mathrm{EP} / \mathrm{SX} / \mathrm{TE}$ & IM & Souza 27 \\
\hline A. auritum Sw. & RU/EP & $\mathrm{CR} / \mathrm{IM}$ & Souza 20 \\
\hline A. cirrhatum Rich ex Willd & $\mathrm{TE}$ & IM & Souza 102 \\
\hline A. claussenii Hieron & $\mathrm{RU} / \mathrm{TE}$ & $\mathrm{CR} / \mathrm{IM}$ & Souza 16 \\
\hline A. flabellulatum Kunze & RU/TE & $\mathrm{CR} / \mathrm{IM}$ & Souza 15 \\
\hline A. geraense (C. Chr.) Sylvestre & RU/TE & CR/IM & Souza 25 \\
\hline A. harpeodes Kunze & $\mathrm{EP} / \mathrm{TE}$ & IM & Souza 125 \\
\hline A. incurvatum Fée & $\mathrm{TE}$ & IM & Souza 222 \\
\hline A. kunzeanum Klotzsch ex Rosenst. & $\mathrm{TE}$ & IM & Souza 202 \\
\hline A. martianum C. Chr. & $\mathrm{RU} / \mathrm{TE}$ & $\mathrm{IM}$ & Souza 69 \\
\hline A. mourai Hieron. & EP & $\mathrm{BM}$ & Roman 92 \\
\hline A. mucronatum C. Presl. & EP & IM & Souza 191 \\
\hline A. oligophyllum Kaulf. & $\mathrm{TE}$ & IM & Souza 72 \\
\hline A. praemorsum $\mathrm{Sw}$. & $\mathrm{TE}$ & CR & Oliveira, 87 \\
\hline A. pseudonitidum Raddi & $\mathrm{RU} / \mathrm{TE}$ & IM & Souza 243 \\
\hline A. radicans $\mathrm{L}$. & $\mathrm{TE}$ & IM & Viana 2032 \\
\hline A. scandicinum Kaulf. & EP & $\mathrm{IM}$ & Souza 225 \\
\hline A. wacketii Rosenst. & RU/TE & IM & Souza 127 \\
\hline \multicolumn{4}{|l|}{ Blechnaceae } \\
\hline Blechnum austrobrasilianum de la Sota & $\mathrm{TE}$ & IM & Abreu 128 \\
\hline B. binervatum subsp. acutum (Desv.) R.M. Tryon \& Stolze & $\mathrm{RU} / \mathrm{SX} / \mathrm{HE}$ & IM & Souza 424 \\
\hline B. brasiliense Desv. & $\mathrm{TE}$ & $\mathrm{BM} / \mathrm{BR}$ & Souza 29 \\
\hline B. cordatum (Desv.) Hieron. & $\mathrm{TE}$ & $\mathrm{CR} / \mathrm{BM} / \mathrm{AA}$ & Souza 198 \\
\hline B. glaziovii Christ & $\mathrm{TE}$ & $\mathrm{BM}$ & Souza 147 \\
\hline B. gracile Kaulf. & $\mathrm{TE}$ & $\mathrm{BM} / \mathrm{IM}$ & Souza 149 \\
\hline B. occidentale $\mathrm{L}$. & $\mathrm{RU} / \mathrm{TE}$ & $\mathrm{BM} / \mathrm{AA}$ & Souza 17 \\
\hline B. organense Brade & $\mathrm{HE}$ & $\mathrm{IM}$ & Souza 614 \\
\hline B. polypodioides Raddi & $\mathrm{TE}$ & $\mathrm{BM} / \mathrm{IM}$ & Ribeiro 177 \\
\hline B. proliferum Rosenst. & $\mathrm{TE}$ & $\mathrm{BM} / \mathrm{IM}$ & Souza 115 \\
\hline B. schomburgki (Klotzsch) C. Chr. & $\mathrm{TE}$ & CR & Viana 1960 \\
\hline
\end{tabular}


Tabela 1. Continuação.

\begin{tabular}{|c|c|c|c|}
\hline Táxon & Hábito & Ambiente & Material Examinado \\
\hline B. $x$ caudatum Cav. & $\mathrm{TE}$ & $\mathrm{BM}$ & Roman 89 \\
\hline Salpichlaena volubilis (Kaulf.) J. Sm. & LI & $\mathrm{IM}$ & Souza 180 \\
\hline \multicolumn{4}{|l|}{ Culcitaceae } \\
\hline Culcita coniifolia (Hook.) Maxon & $\mathrm{TE}$ & IM & Viana 1942 \\
\hline \multicolumn{4}{|l|}{ Cyatheaceae } \\
\hline Alsophila capensis (L.f.) J.Sm. & RU/AR & $\mathrm{BM} / \mathrm{IM}$ & Souza 128 \\
\hline Cyathea atrovirens (Langsd. \& Fisch.) Domin & $\mathrm{AR}$ & $\mathrm{BM}$ & Souza 151 \\
\hline C. corcovadensis (Raddi) Domin & $\mathrm{AR}$ & $\mathrm{BM}$ & Souza 111 \\
\hline C. delgadii Sternb. & $\mathrm{AR}$ & $\mathrm{IM}$ & Roman 25 \\
\hline C. dichromatolepis (Fée) Domin & $\mathrm{AR}$ & $\mathrm{IM}$ & Souza 220 \\
\hline Cyathea gardneri Hook. & $\mathrm{AR}$ & $\mathrm{CR} / \mathrm{BM} / \mathrm{IM}$ & Souza 709 \\
\hline C. glaziovii (Fée) Domin & $\mathrm{AR}$ & IM & Souza 122 \\
\hline C. phalerata Mart. & $\mathrm{AR}$ & $\mathrm{BM} / \mathrm{IM}$ & Souza 65 \\
\hline C. rufa (Fée) Lellinger & $\mathrm{AR}$ & $\mathrm{BM}$ & Souza 634 \\
\hline \multicolumn{4}{|l|}{ Dennstaedtiaceae } \\
\hline Dennstaedtia dissecta (Sw.) T. Moore & $\mathrm{TE}$ & IM & Souza 205 \\
\hline D. globulifera (Poir.) Hieron. & SX & IM & Ribeiro 34 \\
\hline Histiopteris incisa (Thunb.) J. Sm. & $\mathrm{RU} / \mathrm{TE}$ & CR/IM & Salimena 1354 \\
\hline Hypolepis repens (L.) C. Presl & $\mathrm{TE}$ & $\mathrm{BM}$ & Souza 116 \\
\hline Paesia glandulosa (Sw.) Kuhn & $\mathrm{RU} / \mathrm{TE}$ & $\mathrm{CR}$ & Viana 1979 \\
\hline Pteridium arachnoideum (Kaulf.) Maxon & $\mathrm{TE}$ & $\mathrm{BM} / \mathrm{AA}$ & Souza 112 \\
\hline \multicolumn{4}{|l|}{ Dicksoniaceae } \\
\hline Dicksonia sellowiana Hook. & $\mathrm{AR}$ & IM & Souza 229 \\
\hline Lophosoria quadripinnata (J.F. Gmel.) C. Chr. & $\mathrm{TE}$ & $\mathrm{BM}$ & Almeida 755 \\
\hline \multicolumn{4}{|l|}{ Dryopteridaceae } \\
\hline Arachniodes denticulata (Sw.) Ching. & $\mathrm{RU}$ & CR/IM & Souza 241 \\
\hline Ctenitis aspidioides (C.Presl.) Copel. & $\mathrm{TE}$ & $\mathrm{BM} / \mathrm{IM}$ & Souza 114 \\
\hline Didymochlaena truncatula (Sw.) J.Sm. & $\mathrm{TE}$ & IM & Souza 201 \\
\hline Elaphoglossum beaurepairei (Fée) Brade & $\mathrm{TE}$ & IM & Souza 213 \\
\hline E. burchellii (Baker) C. Chr. & $\mathrm{RU} / \mathrm{TE}$ & IM & Souza 34 \\
\hline E. decoratum (Kunze) T. Moore & RU & $\mathrm{BM} / \mathrm{IM}$ & Souza 93 \\
\hline E. edwalii Rosenst. & $\mathrm{TE}$ & IM & Souza 430 \\
\hline E. hybridum (Bory) Brack. & $\mathrm{RU} / \mathrm{TE}$ & $\mathrm{CR} / \mathrm{BM}$ & Souza 755 \\
\hline E. gardnerianum (Kunze ex Fée) T. Moore & EP & IM & Souza 62 \\
\hline E. gayanum (Fée) T. Moore & EP & IM & Souza 46 \\
\hline E. glabellum J. Sm. & RU/EP & IM & Souza 103 \\
\hline E. glaziovii (Fée) Brade & $\mathrm{RU} / \mathrm{SX}$ & IM & Salimena 1290 \\
\hline E. hymenodiastrum (Fée) Brade & RU & CR/IM & Souza 18 \\
\hline E. langsdorffii (Hook. \& Grev.) T. Moore & EP & $\mathrm{IM}$ & Souza 223 \\
\hline E. lingua (C. Presl) Brack. & RU/SX & CR/IM/AA & Viana 2024 \\
\hline
\end{tabular}


Tabela 1. Continuação.

\begin{tabular}{|c|c|c|c|}
\hline Táxon & Hábito & Ambiente & Material Examinado \\
\hline E. longifolium (C. Presl) J. Sm. & EP & IM & Souza 617 \\
\hline E. luridum (Fée) Christ & RU/TE & $\mathrm{CR} / \mathrm{BM} / \mathrm{IM}$ & Souza 179 \\
\hline E. macahense (Fée) Rosenst. & RU & IM & Souza 636 \\
\hline E. pachydermum (Fée) T.Moore & $\mathrm{TE}$ & $\mathrm{CR}$ & Souza 326 \\
\hline E. strictum (Raddi) T.Moore & EP & IM & Souza 425 \\
\hline E. tectum (Humb. \& Bonpl. ex Willd.) T.Moore & $\mathrm{TE}$ & $\mathrm{CR} / \mathrm{BM}$ & Souza 54 \\
\hline Elaphoglossum sp. & $\mathrm{TE}$ & IM & Souza 49 \\
\hline Lastreopsis amplissima (C. Presl) Tindale & $\mathrm{TE}$ & IM & Souza 40 \\
\hline Megalastrum connexum (Kaulf.) A.R.Sm. \& R.C.Moran & $\mathrm{TE}$ & IM & Ribeiro 157 \\
\hline M. crenulans (Fée) A.R. Sm. \& R.C. Moran & $\mathrm{AR}$ & $\mathrm{IM}$ & Souza 724 \\
\hline M. inaequale (Kaulf. ex Link.) A.R.Sm. \& R.C.Moran & $\mathrm{TE}$ & IM & Viana 2029 \\
\hline Mickelia guianensis (Aubl.) R.C. Moran, Labiak \& Sundue & $\mathrm{HE}$ & IM & Souza 204 \\
\hline Olfersia cervina (L.) Kunze & $\mathrm{RU} / \mathrm{HE}$ & IM & Souza 96 \\
\hline Polybotrya speciosa Schott. & $\mathrm{HE}$ & IM & Souza 274 \\
\hline Polystichum montevidense (Spreng.) Rosenst. & $\mathrm{TE}$ & $\mathrm{BM} / \mathrm{IM}$ & Souza 729 \\
\hline Rumohra adiantiformis (G. Forst.) Ching & $\mathrm{RU} / \mathrm{TE}$ & CR & Souza 24 \\
\hline Stigmatopteris caudata (Raddi) C.Chr. & $\mathrm{TE}$ & IM & Souza 192 \\
\hline \multicolumn{4}{|l|}{ Gleicheniaceae } \\
\hline Dicranopteris flexuosa (Schrad.) Underw. & $\mathrm{RU} / \mathrm{TE}$ & $\mathrm{CR} / \mathrm{BM} / \mathrm{AA}$ & Souza 05 \\
\hline D. rufinervis (Mart.) Ching. & $\mathrm{TE}$ & CR/IM & Viana 1965 \\
\hline Sticherus bifidus (Willd.) Ching. & $\mathrm{TE}$ & $\mathrm{CR} / \mathrm{BM} / \mathrm{IM} / \mathrm{AA}$ & Souza 197 \\
\hline S. lanuginosus (Fée) Nakai & $\mathrm{TE}$ & AA & Oliveira 74 \\
\hline S. nigropaleaceus (J.W. Sturm) J.Prado \& Lellinger & $\mathrm{TE}$ & $\mathrm{BM} / \mathrm{AA}$ & Souza 333 \\
\hline \multicolumn{4}{|l|}{ Hymenophyllaceae } \\
\hline Abrodyctium rigidum (Sw.) Ebihara \& Dubuisson & $\mathrm{RU} / \mathrm{TE}$ & IM & Souza 457 \\
\hline Hymenophyllum asplenioides (Sw.) Sw. & EP & IM & Viana 2164 \\
\hline H. elegans Spreng. & $\mathrm{RU}$ & IM & Souza 101 \\
\hline H. fendlerianum J.W. Sturm & RU/EP & IM & Almeida 750 \\
\hline H. fragile (Hedw.) C.V.Morton & $\mathrm{RU}$ & IM & Viana 1992 \\
\hline H. hirsutum (L.) Sw. & $\mathrm{RU} / \mathrm{EP}$ & IM & Souza 210 \\
\hline H. microcarpum Desv. & RU & IM & Viana 2162 \\
\hline H. plumosum Kaulf. & RU & IM & Viana 1912 \\
\hline H. polyanthos (Sw.) Sw. & $\mathrm{RU} / \mathrm{EP} / \mathrm{TE}$ & CR/IM & Souza 41 \\
\hline Polyphlebium angustatum (Carmich.) Ebihara \& Dubuisson & $\mathrm{RU} / \mathrm{EP} / \mathrm{TE}$ & $\mathrm{BM} / \mathrm{IM}$ & Souza 123 \\
\hline P. hymenophylloides (Bosch.) Ebihara \& Dubuisson & $\mathrm{RU}$ & IM & Souza 94 \\
\hline Trichomanes cristatum Kaulf. & $\mathrm{TE}$ & AA & Souza 196 \\
\hline T. pilosum Raddi & $\mathrm{RU} / \mathrm{TE}$ & $\mathrm{BM} / \mathrm{IM}$ & Souza 99 \\
\hline T. polypodioides $\mathrm{L}$. & EP & IM & Souza 717 \\
\hline \multicolumn{4}{|l|}{ Lindsaeaceae } \\
\hline Lindsaea bifida (Kaulf.) Mett. ex Kuhn & $\mathrm{TE}$ & IM & Abreu 209 \\
\hline
\end{tabular}


Tabela 1. Continuação.

\begin{tabular}{|c|c|c|c|}
\hline Táxon & Hábito & Ambiente & Material Examinado \\
\hline L. lancea (L.) Bedd. & RU/EP & IM & Souza 637 \\
\hline \multicolumn{4}{|l|}{ Lomariopsidaceae } \\
\hline Nephrolepis cordifolia (L.) C. Presl & $\mathrm{EP} / \mathrm{TE}$ & CR/IM & Souza 35 \\
\hline \multicolumn{4}{|l|}{ Lycopodiaceae } \\
\hline Huperzia acerosa (Sw.) Holub & EP & IM & Viana 1932 \\
\hline H. flexibilis (Fée) B. Øllg. & EP & IM & Salimena 1368 \\
\hline H. pungentifolia (Silveira) B. Øllg. & $\mathrm{TE}$ & $\mathrm{CR}$ & Souza 216 \\
\hline H. reflexa (Lam.) Trevis. & $\mathrm{TE}$ & $\mathrm{CR} / \mathrm{AA}$ & Viana 1962 \\
\hline Lycopodiella cernua (L.) Pichi-Serm & RU/TE & $\mathrm{CR} / \mathrm{IM} / \mathrm{AA}$ & Souza 28 \\
\hline L. geometra B. Øllg. \& P. G. Windisch & RU & CR & Souza 735 \\
\hline Lycopodium clavatum L. & $\mathrm{TE}$ & $\mathrm{CR} / \mathrm{BM} / \mathrm{IM} / \mathrm{AA}$ & Souza 105 \\
\hline L. thyoides Humb. \& Bonpl. ex Willd. & $\mathrm{TE}$ & $\mathrm{IM}$ & Salimena 2509 \\
\hline \multicolumn{4}{|l|}{ Lygodiaceae } \\
\hline Lygodium volubile Sw. & LI & IM & Souza 33 \\
\hline \multicolumn{4}{|l|}{ Marattiaceae } \\
\hline Danaea geniculata Raddi & $\mathrm{RU} / \mathrm{TE}$ & IM & Souza 208 \\
\hline Eupodium kaulfussii (J. Sm.) J. Sm. & $\mathrm{RU} / \mathrm{TE}$ & IM & Souza 30 \\
\hline \multicolumn{4}{|l|}{ Oleandraceae } \\
\hline Oleandra articulata (Sw.) C. Presl. & RU/SX & IM & Salimena 1286 \\
\hline O. hirta Brack. & RU & CR & Salimena 2757 \\
\hline \multicolumn{4}{|l|}{ Osmundaceae } \\
\hline Osmunda regalis $\mathrm{L}$. & $\mathrm{TE}$ & $\mathrm{BM} / \mathrm{IM}$ & Souza 283 \\
\hline \multicolumn{4}{|l|}{ Polypodiaceae } \\
\hline Alansmia reclinata (Brack.) Moguel \& M. Kessler & EP & IM & Souza 431 \\
\hline Campyloneurum acrocarpon Fée & $\mathrm{RU} / \mathrm{EP} / \mathrm{TE}$ & $\mathrm{CR} / \mathrm{IM}$ & Souza 95 \\
\hline C. austrobrasilianum (Alston) de la Sota & EP & IM & Souza 254 \\
\hline C. decurrens (Raddi) C. Presl & $\mathrm{RU} / \mathrm{TE}$ & IM & Souza 207 \\
\hline C. nitidum (Kaulf.) C. Presl & $\mathrm{RU} / \mathrm{EP} / \mathrm{TE}$ & CR/IM & Souza 37 \\
\hline Ceradenia albidula (Baker) L.E. Bishop & RU & IM & Almeida 748 \\
\hline Cochlidium punctatum (Raddi) L.E.Bishop & EP & IM & Souza 60 \\
\hline C. serrulatum (Sw) L.E.Bishop & $\mathrm{EP} / \mathrm{SX}$ & IM & Souza 59 \\
\hline Grammitis fluminensis Fée & $\mathrm{RU}$ & $\mathrm{IM}$ & Viana 1919 \\
\hline Lellingeria apiculata (Kunze ex Klotzsch) A.R.Sm \& R.C.Moran & EP & CR/IM & Souza 42 \\
\hline L. depressa (C. Chr.) A.R. Sm. \& R.C. Moran & EP & IM & Almeida 768 \\
\hline Leucotrichum organense (Gardner) Labiak & $\mathrm{EP}$ & IM & Souza 438 \\
\hline Melpomene pilosissima (M.Martens \& Galeotti) A.R.Sm. \& R.C.Moran & $\mathrm{RU} / \mathrm{EP} / \mathrm{TE}$ & IM & Souza 50 \\
\hline Microgramma percussa (Cav.) de la Sota & EP & IM & Souza 77 \\
\hline M. squamulosa (Kaulf.) de la Sota & EP & CR/IM & Souza 14 \\
\hline M. tecta (Kaulf.) Alston & EP & IM & Souza 76 \\
\hline M. vaccinniifolia (Langsd. \& Fisch.) Copel. & EP & IM & Valente s.n. (CESJ 49373) \\
\hline
\end{tabular}


Tabela 1. Continuação.

\begin{tabular}{|c|c|c|c|}
\hline Táxon & Hábito & Ambiente & Material Examinado \\
\hline Micropolypodium achilleifolium (Kaulf.) Labiak \& F.B. Matos & EP & $\mathrm{IM}$ & Souza 52 \\
\hline M. gradatum (Baker) Labiak \& F.B.Matos & EP & IM & Viana 1990 \\
\hline Niphidium crassifolium (L.) Lellinger & RU/TE & $\mathrm{CR} / \mathrm{BM}$ & Souza 26 \\
\hline Pecluma chnoophora (Kunze) Salino \& F.C.Assis & $\mathrm{RU} / \mathrm{EP} / \mathrm{SX}$ & $\mathrm{IM}$ & Souza 89 \\
\hline P. pectinatiformis (Lindm.) M.G. Price & $\mathrm{EP} / \mathrm{TE}$ & $\mathrm{CR} / \mathrm{IM}$ & Souza 321 \\
\hline P. recurvata (Kaulf.) M.G. Price & RU/TE & $\mathrm{CR} / \mathrm{IM}$ & Souza 39 \\
\hline P. robusta (Fée) M. Kessler \& A.R. Sm. & $\mathrm{TE}$ & CR/IM & Souza 224 \\
\hline Pecluma sp. 1 & $\mathrm{RU} / \mathrm{TE}$ & $\mathrm{CR} / \mathrm{IM}$ & Souza 32 \\
\hline Pecluma sp. 2 & EP & $\mathrm{IM}$ & Oliveira 56 \\
\hline Phlebodium pseudoaureum (Cav.) Lellinger & $\mathrm{RU} / \mathrm{EP} / \mathrm{TE}$ & $\mathrm{CR} / \mathrm{BM} / \mathrm{IM}$ & Souza 04 \\
\hline Pleopeltis astrolepis (Liebm.) E. Fourn. & EP & IM & Souza 51 \\
\hline P. hirsutissima (Raddi) de la Sota & $\mathrm{RU} / \mathrm{EP} / \mathrm{TE}$ & $\mathrm{CR} / \mathrm{BM} / \mathrm{IM}$ & Salimena 1229 \\
\hline P. macrocarpa (Bory ex Willd.) Kaulf. & EP & $\mathrm{CR} / \mathrm{BM} / \mathrm{IM}$ & Souza 01 \\
\hline P. pleopeltidis (Fée) de la Sota & EP & IM & Souza 437 \\
\hline P. pleopeltifolia (Raddi) Alston & EP & $\mathrm{BM}$ & Souza 307 \\
\hline Serpocaulon catharinae (Langsd. \& Fisch.) A.R.Sm. & $\mathrm{EP} / \mathrm{TE}$ & $\mathrm{CR} / \mathrm{BM} / \mathrm{IM}$ & Salimena 1235 \\
\hline S. fraxinifolium (Jacq.) A.R.Sm. & $\mathrm{EP} / \mathrm{HE}$ & IM & Souza 87 \\
\hline S. latipes (Langsd. \& Fisch.) A.R.Sm. & $\mathrm{RU} / \mathrm{EP} / \mathrm{TE}$ & $\mathrm{CR} / \mathrm{BM} / \mathrm{IM}$ & Souza 66 \\
\hline S. meniscifolium (Langsd. \& Fisch.) A.R. Sm. & $\mathrm{RU} / \mathrm{TE}$ & CR/IM & Souza 06 \\
\hline S. sehnemii (Pic. Serm.) Labiak \& J. Prado & $\mathrm{RU} / \mathrm{TE}$ & CR/AA & Ribeiro 72 \\
\hline S. vacilans (Link.) A.R. Sm. & $\mathrm{EP} / \mathrm{TE}$ & $\mathrm{CR} / \mathrm{IM}$ & Souza 61 \\
\hline Zygophlebia longipilosa (C.Chr.) L.E.Bishop & EP & IM & Souza 217 \\
\hline \multicolumn{4}{|l|}{ Pteridaceae } \\
\hline Adiantopsis radiata (L.)Fée & $\mathrm{TE}$ & CR/IM & Souza 13 \\
\hline Adiantum raddianum C. Presl & $\mathrm{TE}$ & $\mathrm{BM}$ & Souza 148 \\
\hline A. subcordatum $\mathrm{Sw}$. & $\mathrm{TE}$ & $\mathrm{BM} / \mathrm{IM}$ & Abreu 65 \\
\hline Adiantum sp. & $\mathrm{TE}$ & IM & Souza 331 \\
\hline Cheilanthes eriophora (Fée) Mett. & RU & $\mathrm{CR}$ & Souza 10 \\
\hline C. regularis Mett. & $\mathrm{RU} / \mathrm{TE}$ & $\mathrm{IM}$ & Souza 126 \\
\hline Doryopteris collina (Raddi) J. Sm. & $\mathrm{RU} / \mathrm{TE}$ & $\mathrm{CR} / \mathrm{BM} / \mathrm{IM} / \mathrm{AA}$ & Souza 84 \\
\hline D. crenulans (Fée) Christ & $\mathrm{RU} / \mathrm{TE}$ & $\mathrm{CR} / \mathrm{AA}$ & Souza 215 \\
\hline D. lomariacea Kaulf. & $\mathrm{TE}$ & IM & Souza 621 \\
\hline D. majestosa J.C. Yesilyurt & $\mathrm{TE}$ & IM & Souza 698 \\
\hline D. ornithopus (Mett. ex Hook. \& Baker) J.Sm. & $\mathrm{RU} / \mathrm{SX} / \mathrm{TE}$ & $\mathrm{CR}$ & Salimena 1298 \\
\hline D. rosenstockii Brade & $\mathrm{RU} / \mathrm{TE}$ & $\mathrm{CR} / \mathrm{IM}$ & Souza 248 \\
\hline D. rufa Brade & RU & $\mathrm{CR}$ & Almeida 754 \\
\hline D. sagittifolia (Raddi) J.Sm. & $\mathrm{TE}$ & IM & Souza 88 \\
\hline Eriosorus biardii (Fée) A.F. Tryon & RU & $\mathrm{IM}$ & Viana 2006 \\
\hline E. insignis (Kuhn.) A.F. Tryon & $\mathrm{SX} / \mathrm{TE}$ & $\mathrm{BM} / \mathrm{IM}$ & Viana 1963 \\
\hline Pellaea crenata R.M. Tryon & RU & CR & Souza 12 \\
\hline
\end{tabular}


Tabela 1. Continuação.

\begin{tabular}{|c|c|c|c|}
\hline Táxon & Hábito & Ambiente & Material Examinado \\
\hline Pityrogramma calomelanos (L.) Link & RU/TE & CR/AA & Souza 78 \\
\hline Pteris brasiliensis Raddi & $\mathrm{TE}$ & IM & Souza 445 \\
\hline P. decurrens C. Presl & $\mathrm{SX} / \mathrm{TE}$ & IM & Souza 73 \\
\hline P. denticulata Sw. & $\mathrm{TE}$ & IM & Souza 517 \\
\hline P. splendens Kaulf. & $\mathrm{TE}$ & $\mathrm{BM} / \mathrm{IM}$ & Souza 38 \\
\hline P. vittata $\mathrm{L}$. & RU & AA & Souza 82 \\
\hline Vittaria graminifolia Kaulf. & $\mathrm{RU} / \mathrm{TE}$ & IM & Souza 98 \\
\hline V. lineata $(\mathrm{L}.) \mathrm{Sw}$. & RU/EP & IM & Souza 81 \\
\hline \multicolumn{4}{|l|}{ Saccolomataceae } \\
\hline Saccoloma inaequale (Kunze) Mett. & $\mathrm{TE}$ & IM & Souza 629 \\
\hline \multicolumn{4}{|l|}{ Schizaeaceae } \\
\hline Schizaea elegans (Vahl.) Sw. & $\mathrm{TE}$ & IM & Menini Neto 356 \\
\hline \multicolumn{4}{|l|}{ Selaginellaceae } \\
\hline Selaginella decomposita Spring & $\mathrm{RU} / \mathrm{EP} / \mathrm{TE}$ & IM & Souza 70 \\
\hline S. flexuosa Spring & RU/TE & CR/IM & Souza 456 \\
\hline S. macrostachya (Spring) Spring & $\mathrm{RU} / \mathrm{TE}$ & IM & Souza 422 \\
\hline S. marginata (Humb. \& Bonpl. ex Willd.) Spring & $\mathrm{TE}$ & $\mathrm{IM}$ & Salimena 2739 \\
\hline S. muscosa Spring & RU & IM & Ribeiro 42 \\
\hline S. tenuissima Fée & RU & IM & Souza 433 \\
\hline \multicolumn{4}{|l|}{ Thelypteridaceae } \\
\hline Macrothelypteris torresiana (Gaudich.) Ching & $\mathrm{TE}$ & $\mathrm{BM}$ & Souza 113 \\
\hline Thelypteris dentata (Forssk.) E.St. John & $\mathrm{TE}$ & $\mathrm{BM} / \mathrm{IM} / \mathrm{AA}$ & Souza 80 \\
\hline T. interrupta (Willd.) K. Iwats. & $\mathrm{TE}$ & IM & Souza 276 \\
\hline T. longifolia (Desv.) R.M. Tryon & $\mathrm{TE}$ & IM & Roman 04 \\
\hline T. raddii (Rosenst.) Ponce & $\mathrm{TE}$ & $\mathrm{IM}$ & Souza 36 \\
\hline T. regnelliana (C.Chr.) Ponce & $\mathrm{TE}$ & $\mathrm{BM} / \mathrm{IM}$ & Souza 120 \\
\hline T. rivularioides (Fée) Abbiatti & $\mathrm{TE}$ & BM/IM & Souza 117 \\
\hline T. salzmanii (Fée) C.V.Morton & $\mathrm{TE}$ & BR & Souza 107 \\
\hline Thelypteris sp. & $\mathrm{TE}$ & $\mathrm{BM} / \mathrm{IM}$ & Souza 79 \\
\hline \multicolumn{4}{|l|}{ Woodsiaceae } \\
\hline Deparia petersenii (Kunze) Kato & $\mathrm{TE}$ & IM & Souza 74 \\
\hline Diplazium leptocarpon Fée & $\mathrm{RU} / \mathrm{TE}$ & IM & Souza 214 \\
\hline D. lindbergii (Mett.) Christ. & $\mathrm{TE}$ & IM & Ribeiro 156 \\
\hline
\end{tabular}

A maioria das táxons infragenéricos $(42,57 \%)$ tem distribuição geográfica neotropical, corroborando com os trabalhos de Dittrich et. al. (2005) e Melo \& Salino (2006). $\mathrm{Na}$ área também são encontrados 60 espécies endêmicas do Brasil, das quais destacam-se Asplenium geraense citado por Sylvestre (2001) e Salino e Almeida (2008b) como endêmica da Serra do Espinhaço; Cyathea glaziovii, citada por Fernandes (1997) como havendo apenas um registro para o estado de Minas Gerais; Zygophlebia longipilosa é conhecida, em Minas Gerais, apenas na área de estudos e no Parque Estadual do Ibitipoca (Salino et al., dados não publicados), sendo a coleta do presente trabalho, a primeira realizada no estado de Minas Gerais, já que Labiak \& Prado (2005) não citam a ocorrência da espécie para Minas Gerais. A ampliação da distribuição destas espécies reflete a importância de levantamentos 
Tabela 2. Relação de localidades com listagens de pteridófitas publicadas com indicação do número de espécies registradas, tamanho da área, altitude e vegetação dominante em cada localidade. Legenda: estado: MG (Minas Gerais); RJ (Rio de Janeiro); SP (São Paulo); PR (Paraná); Vegetação dominante: FES: Floresta Estacional Semidecidual; FOD: Floresta Ombrófila Densa; FOM: Floresta Ombrófila Mista; FAM: Floresta Aluvial Montana; CS: Campo sujo; CC: Campo Cerrado; CF: Campo Ferruginoso; CQ: Campo quartizítico; CA: Campo de Altitude; CN: Campos Naturais; CR: Campo rupestre; CTR: Costões Rochosos; MGL: Manguezal; RES: Restinga; MC: Mata Ciliar; CX: Caxetais.

\begin{tabular}{|c|c|c|c|c|c|c|}
\hline Localidade & Estado & $\begin{array}{c}\text { Tamanho da área } \\
\text { (ha) }\end{array}$ & Altitude (m.s.m.) & Vegetação dominante & No de espécies & Referência \\
\hline APA-Sul RMBH & MG & 2280 & $790-1420$ & $\mathrm{FES} / \mathrm{CC} / \mathrm{CS} / \mathrm{CF} / \mathrm{CQ}$ & 190 & Figueredo \& Salino 2005 \\
\hline P.E. do Rio Doce & MG & 35973 & $230-515$ & FES & 116 & Melo \& Salino 2002 \\
\hline $\begin{array}{l}\text { Estação Biológica de } \\
\text { Caratinga }\end{array}$ & MG & 880 & $400-680$ & FES & 102 & Melo \& Salino 2002 \\
\hline APA Fernão Dias & MG & não informado & $1000-2068$ & FOD/FOM/FES & 173 & Melo 2003 \\
\hline Cadeia do Espinhaço & MG/BA & & $800-2100$ & CR/CA/CC/FES & 463 & Salino \& Almeida 2008b \\
\hline Serra Negra & MG & 10000 & $900-1698$ & FOD/FES/FAM/CR & 203 & Presente trabalho \\
\hline Reserva Rio das Pedras & $\mathrm{RJ}$ & 1260 & $20-1050$ & não informado & 114 & Mynssen \& Windisch 2004 \\
\hline APA Cairuçu & RJ & 33800 & $0-1320$ & FOD/CTR/MGL/ RES & 115 & Sylvestre 1997b \\
\hline Serra do Cuscuzeiro & SP & não informado & $800-1050$ & FES/CC & 113 & Salino 1996 \\
\hline $\begin{array}{l}\text { Parque Estadual do } \\
\text { Jacupiranga }\end{array}$ & SP & 150000 & $10-1310$ & FOD/RES/CX & 207 & Salino \& Almeida 2008a \\
\hline Parque Ecológico da Klabin & PR & 11196 & 885 & $\mathrm{FES} / \mathrm{FOM} / \mathrm{CN}$ & 121 & Sakagami 2006 \\
\hline P.E. de Vila Velha & PR & 3803,28 & $788-1102$ & $\mathrm{FOM} / \mathrm{CN}$ & 151 & Schwartsburd \& Labiak 2007 \\
\hline P. N. Itatiaia & MG/RJ & 30000 & $500-2789$ & FOD/CA & 135 & Condack 2006 \\
\hline P.E. Itacolomi & MG & 7000 & $660-1760$ & FES/CR & 170 & Rolim 2007 \\
\hline Serra do Caraça & MG & 10187 & $750-2072$ & FES/FOD/CR & 234 & Viveros 2010 \\
\hline PE Ibitipoca & MG & 1923 & $1200-1784$ & CR/FES/FOM & 169 & $\begin{array}{l}\text { Salino et al (dados não } \\
\text { publicados) }\end{array}$ \\
\hline
\end{tabular}

de pteridófitas, uma vez que estes são escassos na região da Serra da Mantiqueira.

De acordo com a Lista Oficial das Espécies da Flora Brasileira Ameaçadas de Extinção (Ministério do Meio Ambiente 2008), na área há duas espécies relacionadas ameaçadas de extinção (Asplenium praemorsum e Dicksonia sellowiana). Já para a Lista Vermelha da Flora Ameaçada para o estado de Minas Gerais (Biodiversitas 2007) há na área 13 espécies classificadas com deficiente de dados, quatro consideradas em perigo, quatro como criticamente em perigo e outras quatro como vulneráveis, além de Alsophila capensis considerada quase ameaçada.

De acordo com Kent \& Coker (1992), duas áreas podem ser consideradas floristicamente similares quando o valor do índice de Sørensen ultrapassa 0,5. Assim, observamos que a Serra Negra mostra-se relacionada à Serra do Caraça $(0,647)$, ao Parque Estadual do Ibitipoca $(0,605)$ e ao Parque Estadual do Itacolomi (0,591) (Fig. 2 e Tab. 3), isso, provavelmente, deve-se às áreas apresentarem fitofisionomias parecidas compostas principalmente por campos rupestres entremeados a formações florestais.

Analisando de maneira geral o dendrograma do Coeficiente de Sørensen (Fig. 2) e a matriz de similaridade (Tab. 3), percebemos que, provavelmente, as fitofisionomias influen- ciam na distribuição desse grupo de plantas, pois observamos a formação de cinco grupos entre as áreas. O grupo formado pelo Parque Estadual do Jacupiranga, APA Cairuçu e Reserva Rio das Pedras, apresenta como fitofisionomias dominantes Restinga e Floresta Ombrófila Densa; outro grupo é formado pela Estação Biológica de Caratinga e Parque Estadual do Rio Doce, que compartilham a mesma fitofisionomia, Floresta Estacional Semidecidual, além de serem áreas próximas geograficamente. Na Figura 2 observamos ainda um conjunto formado pela Serra do Cuscuzeiro e a APA Sul de Belo Horizonte, onde provavelmente a existência de Cerrado e Floresta Estacional Semidecidual relaciona tais áreas. O agrupamento formado pelo Parque Estadual de Vila Velha e o Parque Ecológico da Klabin pode também ser explicado pela fitofisionomia, formações florestais com campos naturais, e distância geográfica. Finalmente a APA Fernão Dias mostra-se mais relacionada ao grupo anterior, porém com uma baixa correlação $(0,514)$, provavelmente devido à composição específica dessas áreas, uma vez que a APA Fernão Dias apresenta formação vegetacional diferente das demais.

O presente trabalho revela uma elevada riqueza de pteridófitas na área, indicando assim a importância de estudos desta natureza como ferramenta na conservação e manejo das espécies de pteridófitas em Minas Gerais e no Brasil. 


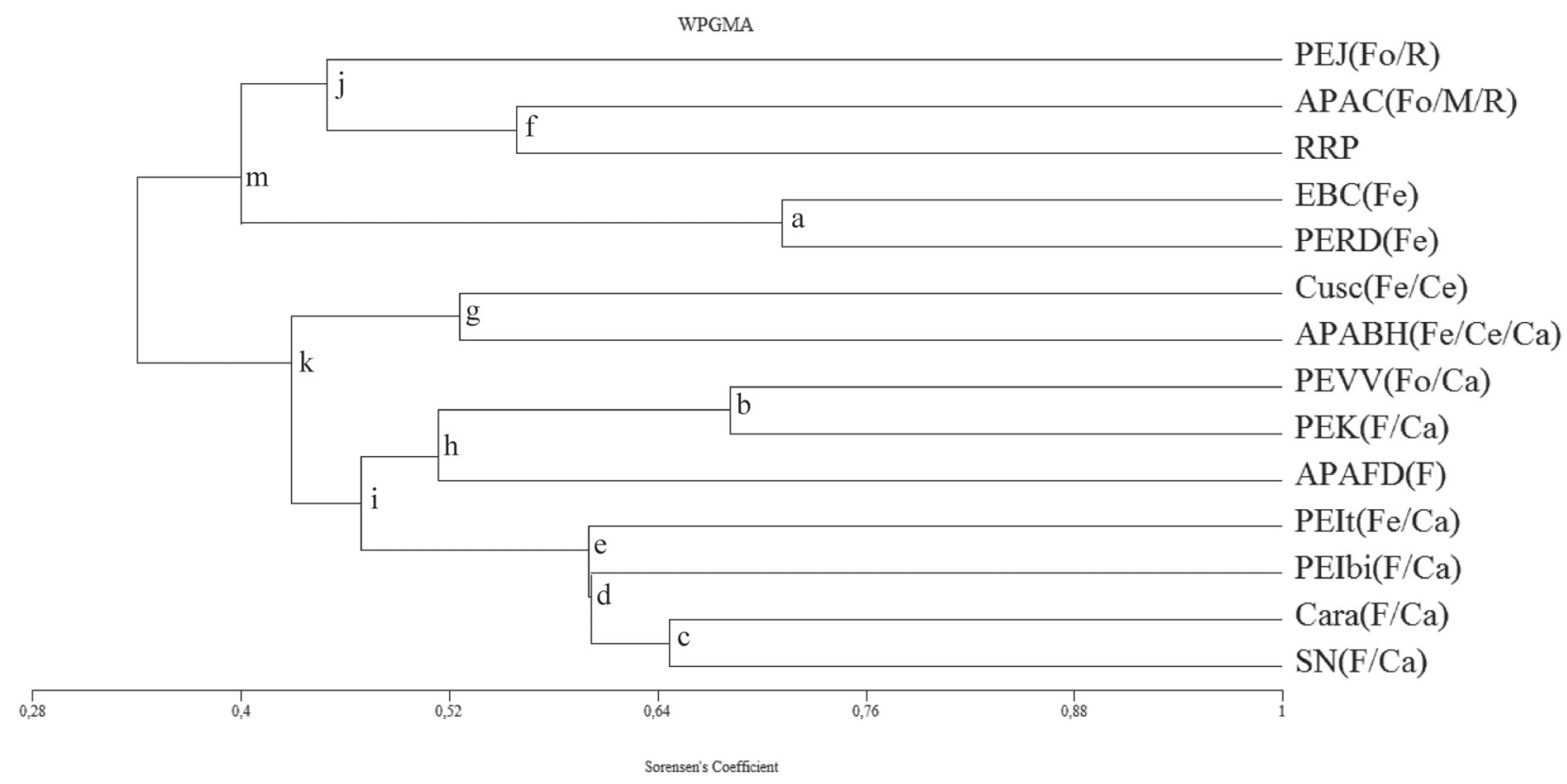

Figura 2. Dendrograma de similaridade de espécies de pteridófitas entre 14 áreas da Floresta Atlântica, utilizando índice de Sørensen e algoritimo WPGMA. SN (Serra Negra), APABH (APA Sul de Belo Horizonte), PERD (Parque Estadual do Rio Doce), EBC (Estação Biológica de Caratinga), APAFD (APA Fernão Dias), RRP (Reserva Rio das Pedras), APAC (APA Cairuçu), Cusc(Serra do Cuscuzeiro), PEJ (Parque Estadual de Jacupiranga), PEK (Parque Ecológico da Klabin), PEVV (Parque Estadual de Vila Velha), PEIt (Parque Estadual do Itacolomi), Cara (Serra do Caraça), PEIbi (Parque Estadual do Ibitipoca). F (Floresta Ombrófila e Estacional), $\mathrm{Ca}$ (Campo), Fe (Floresta Estacional), Ce (Cerrado), Fo (Floresta Ombrófila), M (Manguezal), R (Restinga). Letras minúsculas referem-se aos valores do índice para os nós: $\mathrm{a}=0,712 ; \mathrm{b}=0,682 ; \mathrm{c}=0,647 ; \mathrm{d}=0,602 ; \mathrm{e}=0,6 ; \mathrm{f}=0,559 ; \mathrm{g}=0,526 ; \mathrm{h}=0,514 ; \mathrm{i}=0,469 ; \mathrm{j}=0,45 ; \mathrm{k}=0,429 ; \mathrm{m}=0,4$.

Tabela 3. Matriz de similaridade de Pteridófitas entre 14 áreas da Floresta Atlântica, utilizando índice de Sørensen e algoritimo WPGMA. Em negrito destacam-se os maiores valores. SN (Serra Negra), APABH (APA Sul de Belo Horizonte), PERD (Parque Estadual do Rio Doce), EBC (Estação Biológica de Caratinga), APAFD (APA Fernão Dias), RRP (Reserva Rio das Pedras), APAC (APA Cairuçu), Cusc (Serra do Cuscuzeiro), PEJ (Parque Estadual de Jacupiranga), PEK (Parque Ecológico da Klabin), PEVV (Parque Estadual de Vila Velha), PEIt (Parque Estadual do Itacolomi), Cara (Serra do Caraça), PEIbi (Parque Estadual do Ibitipoca). F (Floresta Ombrófila e Estacional), Ca (Campo), Fe (Floresta Estacional), Ce (Cerrado), Fo (Floresta Ombrófila), M (Manguezal), R (Restinga).

\begin{tabular}{|c|c|c|c|c|c|c|c|c|c|c|c|c|c|c|}
\hline & $\begin{array}{c}\mathrm{SN} \\
(\mathrm{F} / \mathrm{Ca})\end{array}$ & $\begin{array}{c}\mathrm{APABH} \\
(\mathrm{Fe} / \mathrm{Ce} / \mathrm{Ca})\end{array}$ & $\begin{array}{l}\text { PERD } \\
(\mathrm{Fe})\end{array}$ & $\begin{array}{l}\mathrm{EBC} \\
(\mathrm{Fe})\end{array}$ & $\begin{array}{l}\text { APAFD } \\
(\mathrm{F})\end{array}$ & RRP & $\begin{array}{c}\mathrm{APAC} \\
(\mathrm{Fo} / \mathrm{M} / \mathrm{R})\end{array}$ & $\begin{array}{l}\text { Cusc } \\
(\mathrm{Fe} / \mathrm{Ce})\end{array}$ & $\begin{array}{c}\mathrm{PEJ} \\
(\mathrm{Fo} / \mathrm{R})\end{array}$ & $\begin{array}{c}\text { PEK } \\
(\mathrm{F} / \mathrm{Ca})\end{array}$ & $\begin{array}{l}\text { PEVV } \\
(\mathrm{Fo} / \mathrm{Ca})\end{array}$ & $\begin{array}{c}\text { PEIt } \\
(\mathrm{Fe} / \mathrm{Ca})\end{array}$ & $\begin{array}{c}\text { Cara } \\
(\mathrm{F} / \mathrm{Ca})\end{array}$ & $\begin{array}{l}\text { PEIbi } \\
(\mathrm{F} / \mathrm{Ca})\end{array}$ \\
\hline $\mathrm{SN}(\mathrm{F} / \mathrm{Ca})$ & 1 & & & & & & & & & & & & & \\
\hline $\mathrm{APABH}(\mathrm{Fe} / \mathrm{Ce} / \mathrm{Ca})$ & 0,491 & 1 & & & & & & & & & & & & \\
\hline $\operatorname{PERD}(\mathrm{Fe})$ & 0,277 & 0,364 & 1 & & & & & & & & & & & \\
\hline $\mathrm{EBC}(\mathrm{Fe})$ & 0,3 & 0,406 & 0,712 & 1 & & & & & & & & & & \\
\hline $\operatorname{APAFD}(\mathrm{F})$ & 0,555 & 0,438 & 0,194 & 0,216 & 1 & & & & & & & & & \\
\hline RRP & 0,328 & 0,376 & 0,409 & 0,4 & 0,245 & 1 & & & & & & & & \\
\hline $\mathrm{APAC}(\mathrm{Fo} / \mathrm{M} / \mathrm{R})$ & 0,371 & 0,333 & 0,391 & 0,371 & 0,275 & 0,559 & 1 & & & & & & & \\
\hline $\mathrm{Cusc}(\mathrm{Fe} / \mathrm{Ce})$ & 0,39 & 0,526 & 0,37 & 0,398 & 0,412 & 0,341 & 0,3 & 1 & & & & & & \\
\hline $\mathrm{PEJ}(\mathrm{Fo} / \mathrm{R})$ & 0,473 & 0,46 & 0,408 & 0,408 & 0,405 & 0,436 & 0,463 & 0,397 & 1 & & & & & \\
\hline $\mathrm{PEK}(\mathrm{F} / \mathrm{Ca})$ & 0,444 & 0,378 & 0,296 & 0,282 & 0,472 & 0,287 & 0,318 & 0,462 & 0,426 & 1 & & & & \\
\hline $\operatorname{PEVV(Fo/Ca)~}$ & 0,5 & 0,432 & 0,242 & 0,26 & 0,555 & 0,259 & 0,324 & 0,409 & 0,483 & 0,682 & 1 & & & \\
\hline $\mathrm{PEIt}(\mathrm{Fe} / \mathrm{Ca})$ & 0,591 & 0,549 & 0,282 & 0,292 & 0,509 & 0,275 & 0,299 & 0,407 & 0,397 & 0,39 & 0,508 & 1 & & \\
\hline $\operatorname{Cara}(\mathrm{F} / \mathrm{Ca})$ & 0,647 & 0,526 & 0,254 & 0,28 & 0,441 & 0,22 & 0,263 & 0,292 & 0,41 & 0,332 & 0,426 & 0,61 & 1 & \\
\hline PEIbi(F/Ca) & 0,605 & 0,422 & 0,18 & 0,179 & 0,496 & 0,232 & 0,269 & 0,304 & 0,337 & 0,353 & 0,475 & 0,599 & 0,598 & 1 \\
\hline
\end{tabular}




\section{Agradecimento}

À FAPEMIG, pela concessão de auxílio ao projeto "Estudos Florísticos na Serra Negra, Minas Gerais" (CRA 1891/06 e CRA 1810-5.02/07) e ao Sr. Ceslau Gomes Ferreira Freitas e à comunidade da Vila do Funil que permitiram a realização deste trabalho em suas propriedades. À doutoranda Luciana C. Mello pelas identificações de Elaphoglossum. À mestre Nara F. O. Mota pela ajuda e dicas no início do trabalho, ao Mestrando Márcio Malafaia Filho pela confecção do mapa e a toda a equipe do Herbário Leopoldo Krieger em especial a equipe do projeto Flora da Serra Negra por toda ajuda que deram. Ao CNPq pela bolsa produtividade concedida à Alexandre Salino.

\section{Referências bibliográficas}

Assis, F.C. \& Salino, A. 2011. Dennstaedtiaceae (Polypodiopsida) no estado de Minas Gerais, Brasil. Rodriguesia 62(1): 11-33.

Boldrin, A.H.L. \& Prado, J. 2007. Pteridófitas terrestres e rupícolas do Forte dos Andradas, Guarujá, São Paulo, Brasil. Boletim de Botânica da Universidade de São Paulo 25(1): 1-69.

Brade, A.C. 1942. Excursão à Serra do Caparaó. Rodriguésia 15: 87-92.

Brade, A.C. 1948. Contribuição para o conhecimento da flora do Estado do Espírito Santo (I. Pteridophyta). Rodriguésia 10: 25-56.

Brade, A.C. 1949. Relatório de uma excursão ao município de Passa Quatro, estado de Minas Gerais. Rodriguésia 23: 133-142.

Camargo, R.F.N. 1983. Pteridófitas rupícolas e saxícolas do Sudeste de Minas Gerais (Brasil). Dissertação de Mestrado. Museu Nacional, Rio de Janeiro.

Carvalho, I.R. 1982. O gênero Anemia Sw. nos Campos rupestres da Cadeia do Espinhaço no Estado de Minas Gerais. Dissertação de Mestrado. Instituto de Biociências (UNESP) Campus Rio Claro, São Paulo.

Condack, J.P.S. 2006. Pteridófitas ocorrentes na região alto Montana do Parque Nacional do Itatiaia: análise florística e estrutural. Dissertação de Mestrado. Jardim Botânico do Rio de Janeiro, Rio de Janeiro.

Dittrich, V.A.O.; Waechter, J.L. \& Salino, A. 2005. Species richness of pteridophytes in a montane Atlantic rain forest plot of Southern Brazil. Acta Botanica Brasilica 19(3): 519-525.

Drummond, G.M; Machado, A.B.M.; Martins, C.S.; Mendonça, M.P. \& Stehmann, J.R. 2008. Listas vermelhas das espécies da fauna e da flora ameaçadas de extinção em Minas Gerais. 2 ed. Belo Horizonte, Fundação Biodiversitas.

Drummond, G.M.; Martins, C.S.; Machado, A.B.M.; Sebaio, F.A. \& Antonini, Y. 2005. Biodiversidade em Minas Gerais: um atlas para sua conservação. Fundação Biodiversitas, Belo Horizonte, MG.

Fernandes, I. 1997. Taxonomia e fitogeografia de Cyatheaceae e Dicksoniaceae nas regióes sul e sudeste do Brasil. Tese de Doutorado. Instituto de Biociências da Universidade de São Paulo, São Paulo.

Figueiredo, J.B. \& Salino, A. 2005. Pteridófitas de quatro Reservas Particulares do Patrimônio Natural ao Sul da Região Metropolitana de Belo Horizonte, Minas Gerais, Brasil. Lundiana 6(2): 83-94.

Filgueiras, T.S.; Nogueira, P.E.; Brochado, A.L. \& Guala, G.F. 1994. Caminhamento: um método expedito para levantamentos florísticos qualitativos. Cadernos de Geociências 12: 39-43.

Grayum, M.H. \& Churchill, H.W. 1987. An introduction to the pteridophyte flora of Finca La Selva, Costa Rica. American Fern Journal 77: 73-89.

Joly, A.J., Leitão Filho, H.F. \& Silva, S.M. 1991. O patrimônio florístico. Pp. 97-125. In: Cecchi, J.C. \& Soares, M.S.M. (Coords.). Mata Atlântica. Fundação SOS Mata Atlântica. São Paulo, Editora Index.

Kent, M. \& Coker, P. 1992. Vegetation description and analysis. London, John Wiley \& Sons.

Köppen, W. 1931. Grundriss der Klimakunde. Berlin, Walter de Gruyter.
Kramer, K.U. \& Green, P.S. 1990. Pteridophytes and Gymnosperms. In: Kubitzki, K. The Families and Genera of Vascular Plants. v. 1. New York, Springer-Verlag.

Labiak, P.H. \& Prado, J. 2005. As espécies de Terpsichore A.R. Sm. e Zygophlebia L. E. Bishop (Grammitidaceae) do Brasil. Acta Botanica Brasilica 19(4): 869-889.

Magurran, A.E. 2004. Measuring Biological Diversity. Oxford, Blackwell Science.

Melo, L.C.N. \& Salino, A. 2002. Pteridófitas de duas áreas de floresta da Bacia do Rio Doce no Estado de Minas Gerais, Brasil. Lundiana 3(2): 129-139.

Melo, L.C.N. \& Salino, A. 2007. Pteridófitas em fragmentos florestais da APA Fernão Dias, Minas Gerais, Brasil. Rodriguésia 58(1): 207-220.

Menini Neto, L.; Matozinhos, C.N.; Abreu, N.L.; Valente, A.S.M.; Antunes, K.; Souza, F.S.; Viana, P.L. \& Salimena, F.R.G. 2009. Flora vascular não-arbórea de uma floresta de grota na Serra da Mantiqueira, Zona da Mata de Minas Gerais, Brasil. Biota Neotropica 9(4): 1-13.

Minestério Meio do Ambiente. Lista Nacional das Espécies da Flora Brasileira Ameaçada de Extinção. Anexo à Instrução Normativa No 6, DE 23 de Setembro de 2008 do Ministério do Meio Ambiente, 23 Setembro 2008. Disponivel em: <http://www.mma.gov.br/ estruturas/179/_arquivos/179_05122008033615.pdf $>$.

Moran, R.C. 1995. The importance of montains to pteridophytes, with emphasis on neotropical montane forests. Pp. 359-363. In: Churchill, S.P.; Balslev, H.; Forero, E. \& Luteyn, J.L. (Eds.) Biodiversity and conservation of neotropical montane forests. The New York Botanical Garden.

Myers, N.; Mittermeier, R.A.; Mittermeier, C.G.; Fonseca, G.A.B. \& Kent, J. 2000. Biodiversity hotspots for conservation priorities. Nature. 403: $853-858$

Mynssen, C.M. \& Windisch, P.G. 2004. Pteridófitas da Reserva de Rio das Pedras, Mangaratiba, RJ. Rodriguésia 55(85): 125-156.

Novelino, R.F. \& José Emílio Zanzirolani de Oliveira. 1999. Flora do Parque Estadual do Ibitipoca Minas Gerais, Brasil: Elaphoglossaceae (Pteridophyta). Revista Meio Ambiente Em Debate Ibama, Brasília 27:7-35.

Page, C. N. 1979. The diversity of ferns. An ecological perspective. Pp.1056. In: Dyer, A.F. The experimental biology of the ferns. London, Academic Press.

Pichi Sermolli, R.E.G. 1996. Authors of scientific names in Pteridophyta. Royal Botanic Gardens, Richmond.

Prado, J. 1992. Flora da Serra do Cipó, Minas Gerais. Pteridaceae Cheilanthoideae. Boletim de Botânica da Universidade de São Paulo 13: 141-159.

Prado, J. 1997. Flora da Serra do Cipó, Minas Gerais. Pteridaceae Adiantoideae e Taenitoideae. Boletim de Botânica da Universidade de São Paulo 16: 115-118.

Prado, J. \& Labiak, P.H. 2003. Pteridófitas. In: Pirani, J.R.; Mello-Silva, R. \& Giulietti, A.M. (Orgs.). Flora de Grão Mogol, Minas Gerais. Boletim de Botânica da Universidade de São Paulo 21: 25-47.

Prado, J., Sylvestre, L. 2011. Pteridófitas in Lista de Espécies da Flora do Brasil. Jardim Botânico do Rio de Janeiro. (http://floradobrasil.jbrj. gov.br/2011/FB000007).

Prado, J. \& Windisch, P. G. 1996. Flora da Serra do Cipó, Minas Gerais. Dennstaedtiaceae. Boletim de Botânica da Universidade de São Paulo 15: 83-88.

Rolim, L.B. 2007. Pteridófitas do Parque Estadual do Itacolomi, Minas Gerais, Brasil. Dissertação de Mestrado. Universidade Federal de Minas Gerais, Belo Horizonte.

Rolim, L.B. \& Salino, A. 2008. Polypodiaceae Bercht \& Presl (Polypodiopsida) no Parque Estadual do Itacolomi, MG, Brasil. Lundiana 9: 83-106.

Sakagami, C.R. 2006. Pteridófitas do Parque Ecológico da Klabin, Telêmaco Borba, Paraná, Brasil. Dissertação de Mestrado. Universidade Federal do Paraná, Curitiba.

Salino, A. 1996. Levantamento das pteridófitas da Serra do Cuscuzeiro, Analândia, SP, Brasil. Revista Brasileira de Botânica 19(2): 173-178.

Salino, A. \& Almeida, T.E. 2008a. Pteridófitas do Parque Estadual do Jacupiranga, SP, Brasil. Acta Botanica Brasilica 22(4): 983-991.

Salino, A. \& Almeida, T.E. 2008b. Diversidade e conservação das pteridófitas na Cadeia do Espinhaço, Brasil. Megadiversidade 4: 196-216. 
Salino, A. \& Almeida, T.E. 2009. Pteridófitas. Pp.19-25. In: Stehmann, J.R.; Forzza, R.C.; Salino, A.; Sobral, M.; Costa, D.P. \& Kamino, L.H.Y. 2009. Plantas da Floresta Atlântica. Jardim Botânico do Rio de Janeiro, Rio de Janeiro.

Salino, A. \& Garcia, P.A. 2008. Dryopteridaceae (Polypodiopsida) no Estado de Minas Gerais, Brasil. Lundiana 9: 3-27.

Santos, M.G. \& Sylvestre, L.S. 2006. Aspectos florísticos e econômicos das pteridófitas de um afloramento rochoso do Estado do Rio de Janeiro, Brasil. Acta Botanica Brasilica 20(1): 115-124.

Santos, M.G., Sylvestre, L.S. \& Araujo, D.S.D. 2004. Análise florística das pteridófitas do Parque Nacional da Restinga de Jurubatiba, Rio de Janeiro, Brasil. Acta Botanica Brasilica 18(2): 271-280.

Schwartsburd, P.B. \& Labiak, P.H. 2007. Pteridófitas do Parque Estadual de Vila Velha, Ponta Grossa, Paraná, Brasil. Hoehnea 34(2): 159-209.

Silva, A.T. 1989. Pteridófitas. Pp. 33-34. In: Bononi, V.L.R. \& Fidalgo, O (Eds.). Técnicas de coleta, preservação e herborização de material botânico. Instituto de Botânica, São Paulo.

Smith, A.R.; Pryer, K.M.; Schuettpelz, E.; Korall, P.; Schneider, H. \& Wolf, P.G. 2006a. A classification for extant ferns. Taxon 55(3): 705-731.

Sokal, R.R. \& Michener, C.D. 1958. A statistical method for evaluating systematic relationship. University of Kansas Society Bulletim 38: 1409-1438.

Sylvestre, L. S. 1997a. Pteridófitas da Reserva Ecológica Macaé de Cima. Pp. 40-52. In: Lima, H.C. \& Guedes-Bruni, R.R. Serra de Macaé de
Cima: Diversidade Florística e Conservação em Mata Atlântica. Rio de Janeiro, Jardim Botânico do Rio de Janeiro.

Sylvestre, L. S. 1997b. Pteridophyta. Pp. 44-49. In: Marques, M.C.M.; Vaz, A.S.F. \& Marquete, R. Mapeamento da cobertura vegetal e listagem das espécies ocorrentes na Área de Proteção Ambiental de Cairuçu, Município de Parati, Rio de Janeiro. Jardim Botânico do Rio de Janeiro/Ministério do Meio Ambiente, Rio de Janeiro.

Sylvestre, L.S. 2001. Revisão das espécies da família Aspleniaceae A. B. Frank ocorrentes no Brasil. Tese de Doutorado. Universidade de São Paulo, São Paulo.

Tryon, R. M. 1972. Endemic areas and geographic speciation in tropical american ferns. Biotropica 4(3): 121-131.

Tryon, R. M. \& Tryon, A. F. 1982. Ferns and Allied Plants, with Special Reference to Tropical America. New York, Springer Verlag.

Viveros, R. S. 2010. Pteridófitas da Serra do Caraça, Minas Gerais, Brasil. Dissertação de Mestrado. Universidade Federal de Minas Gerais, Belo Horizonte.

Windisch, P.G. \& Prado, J. 1990. Flora da Serra do Cipó, Minas Gerais. Cyatheaceae. Boletim de Botânica da Universidade de São Paulo 12:7-13.

Windisch, P.G. 1992a. Flora da Serra do Cipó, Minas Gerais. Hymenophyllaceae. Boletim de Botânica da Universidade de São Paulo 13: 133-139. 